\title{
Content-Based Tweets Clustering and Analysis
}

\author{
Jahiruddin \\ Department of Computer Science \\ Jamia Millia Islamia (Central University) \\ New Delhi-110025, India \\ E-mail: jahir.jmi@gmail.com
}

\begin{abstract}
Event-based social network analysis is an important task for monitoring the potential threats to the security of a nation and identifying various trends that are popular among the people. In this paper, we propose content-based tweets clustering and analysis method, which aims to cluster tweets based on the events represented by them. The proposed method starts with modeling tweets into a similarity graph (aka social network), in which each node represent a tweet and an edge connecting a node-pair represents the degree of similarity between the tweets represented by them. For social graph generation, each node is represented as a feature vector which is generated using Latent Dirichlet Allocation (LDA) from the respective tweet and edge weight is determined as the similarity between the nodes. Finally, the generated social graph is partitioned into a number of clusters (sub-graphs) using Markov Clustering (MCL) algorithm, where each sub-graph represent an event. We have generated a data set of $\mathbf{5 0 0 0}$ tweets related to four different events - Uri attacks, Delhi assembly election, Union budget 2015, and Israel-Gaza conflict to evaluate the proposed method. The experimental results are encouraging, showing high accuracy in grouping tweets based on their contents. We have also performed a comparative analysis of the Cosine similarity and Euclidean distance based similarity graph generation, and it is found that the Cosine similarity yields better results than the Euclidian distance measure.
\end{abstract}

Keywords- Twitter data analysis; Similarity graph generation; Key term extraction; Graph clustering; Event classification.

\section{INTRODUCTION}

The recent advancements in Web technologies has motivated young generations to use online social networks like Facebook, Twitter, Instagram, Tumbler etc. They use the online social network for various purposes, including updating of events and sharing of the new and useful information. As a result, presently online social network becomes a powerful tool among internet users to share their views with other internet users. One of the fastest-growing online social media is Twitter. It is a popular social media website which allows users to share their views in the form of tweets that is a short message up to 140 characters long. Recently the Twitter extended its message length from 140 characters to 280 characters. Besides tweeting a short message, Twitter is also used for marketing, election campaign, and for spreading news. In addition to these, it is also used by the social media users to express their opinions on important social and political issues occurred around their locality or world. Tweet analysis for detecting emerging issues and trends are considerable interest to various stakeholders, including private companies, security agencies, and governments.

The tweet analysis is a technically challenging task, due to its unstructured nature and use of the informal natural languages. There are millions of tweets on a number of topics are generated every day by a large number of users. The grouping of the tweets on the basis of topics or events is a ground challenging task in analyzing Twitter data. A real life event may be conceptualized using key terms which are embedded in the tweets. For example, "Uri attacks" event can be conceptualized using the key terms attack, terrorist, solders, uri etc., whereas vote, election, political-party, etc. can be used to conceptualize the "Delhi assembly election" event.

In this paper, we use the graph model to analyze Twitter data using the similarity graph generation followed by clustering of these similarity graph. Tweets are cleaned and then tokenized using NLP techniques to generate candidate terms and these candidate keys are ranked using Latent Dirichlet Allocation (LDA) method to get top ranked key terms which are used to convert each tweet into a feature vector. Thereafter, Cosine similarity and Euclidian distance methods are used to generate similarity graph of the underline tweets. Finally, the similarity graph is clustered using MCL algorithm to partition it into a number of sub-graphs (cluster), each sub-graph represent a set of tweets related to a particular event. This paper is a substantially extended version of one of our previous works [1]. In this paper, in addition to presenting the proposed tweets analysis method in a broader perspective, we have presented a comparative analysis of the Cosine similarity and Euclidian distance measures in social graph generation and event identification. 
The remaining part of the paper is organized as follows. Section 2 presents a brief review of the twitter data analysis techniques. In section 3 we presented the basis of mathematics used in similarity graph generation. The proposed tweets clustering method is presented in sections 4 . The experimentally evaluation of the proposed method is presented in section 5. Finally, section 6 concludes the paper with future directions of work.

\section{RELATED WORKS}

Twitter is a popular social media website among Internet users. Millions of messages, on different topics, are posted by a large numbers of users daily on this social media website. In case of Twitter, these message is called tweet. The authors of these tweets share their opinions on real life events and discuss different issues of the society. Recently, a large number of literatures have proposed methods to analyze social network data, especially the Twitter data, for various purposes $[2,3,4,5,6,7]$. In [8], the authors used the predictive power of social media data to identify the conflicting in US election 2010 using sentiment analysis methods. Cheong and Lee [9] used the Self Organizing Map (SOM) to identify interesting pattern in Iran election 2009. Akcora et al. [10] developed a tool for internet users to view the important news stories and search the article of their interest on the Web. In [11], the authors proposed a method that may be used in stock market prediction. In [12], the authors assessed whether there are an association between popular events and sentiment strength.

The influence tracking on the social media is another important task. This may be used in some real applications like marketing of the online products, country election, as influential users may change the mind of large number of users as they play an important role in the society. Cha et al. [13] analyzed the social network graph using structural features like in-degree of vertices, re-tweets, and user mentions. Their method helps in influence tracking dynamically across topic and time. Willis et al. [14] uses ageing factor analysis of the tweets and response types to determine the influence of their individual tweets. They reported that BBC corporate account @BBCSport play important role in key actor analysis that is used in influential tracking.

Another important research area in Twitter data mining is opinion mining and sentiment analysis. In [15] Pak and Paroubek, proposed a system to get the sentiment of a tweet using linguistic analysis. In [16], the authors proposed multi-nominal naive Bayes classifier which assigns positive or negative sentiment class value to tweets. They compared a number of classifiers and reported that their algorithm outperforms the other approaches. Spencer and Uchyigit [17] developed a sentiment analysis tool for tweeter data that classify the tweets into three classes - positive, negative, and objective class. Go et al. [18] proposed a system for sentiment classification on Twitter data. At the place of explicit rating such as star rating, they use the emoticons :) and :( for indentifying the positive and negative tweets. Due to binary classification and non-consideration of objective text this system is highly limited. They reported that the best result classification of tweets is achieved by using unigrams and bigrams in combined.

The event identification is another key research area in the field of Social media data mining. Becker et al. [19] proposed a framework to identify an event in a set of social media documents. They produced high quality clusters of similar social media documents using object similarity metric approach. They reported that their technique, which used similarity metric, gives better performance over traditional document clustering approaches that consider only text-based similarity. In [20], the authors presented a method for real-time events identification. This method used the tweets content like key terms and their context, and number of such key terms for detecting earthquake event. In [21], the authors developed a binary classifier that classifies the tweets into sets of event tweets and non-event tweets. For classification of tweets, they have used social, temporal, and topical features of the tweets along with the some of the Twitter-centric features. In contrast to classification of tweets, in this paper we consider content-based tweets analysis as a clustering problem which partition the set of tweets into a number of clusters based on number of events are described by them. A detailed review of the state-of-the arts in social network mining and its applications is presented in [22].

\section{PRELIMINARIES FOR SIMILARITY GRAPH GENERATION}

In this section, we present the mathematical basis for similarity graph generation. Started with the basic concept of inner product and vector norm, the cosine similarity and Euclidian distance based similarity is presented in subsequent subsections.

\section{A. Inner Product and Vector Norms}

Inner product (aka dot product) of vector $u$ and $v$ is denoted by $\langle u, v\rangle$ is a scalar quantity. The inner product of $n$-dimensional vectors $u=\left(u_{1}, u_{2}, \ldots, u_{n}\right)^{T}$ and $v=\left(v_{1}, v_{2}, \ldots, v_{n}\right)^{T}$ in vector space $\mathcal{R}^{\mathrm{n}}$ is defined using equation $1[23]$.

$$
\langle u, v\rangle=u . v=u_{1} v_{1}+u_{2} v_{2}+\cdots+u_{n} v_{n}=\sum_{i=1}^{n} u_{i} v_{i}
$$


The vector norm of an $n$-dimensional vector $u$ in vector space $\mathcal{R}^{\mathrm{n}}$ is a function that assign a non negative real number to the vector. The inner product of vector with itself gives square of the vector norm, but every vector norm is not determined using inner product [23]. The vector norm of an n-dimensional vector $u=\left(u_{1}, u_{2}, \ldots\right.$, $\left.u_{n}\right)^{T}$ is denoted by $\|u\|$ and can be define using equation 2 .

$$
\|u\|=\sqrt{\langle u, u\rangle}
$$

There are a number of vector norms but most popular vector norms are $\mathrm{L}_{1}$-norm (aka Manhattan norm) and $\mathrm{L}_{2}$-norm (aka Euclidean norm) [24]. The $\mathrm{L}_{1}$-norm of vector $u$ is obtained by adding the absolute value of its components and defined using equation 3 . Whereas, $\mathrm{L}_{2}$-norm of vector $u$ is positive square root of the sum of square of its components and is defined using equation 4. The Euclidian norm of a vector $u$ gives the length of the vector.

$$
\begin{gathered}
\|u\|_{1}=\sum_{i=1}^{n}\left|u_{i}\right| \\
\|u\|_{2}=\sqrt{\sum_{i=1}^{n} u_{i}^{2}}
\end{gathered}
$$

\section{B. Cosine Similarity}

Let vector $u=\left(u_{1}, u_{2}, \ldots, u_{n}\right)^{T}$ and $v=\left(v_{1}, v_{2}, \ldots, v_{n}\right)^{T}$ are two $n$-dimensional vectors in vector space $\mathcal{R}^{\mathrm{n}}$, then cosine similarity between these two vectors should be a real number between -1 and 1 is the cosine of angle between these two vectors and calculated using equation 5 . Since in our case, the value of each tweet vector components should be either 0 or 1 so the cosine similarity value between each tweet-pair should be in range from 0 to 1 .

$$
\text { similarity }=\operatorname{cosine}(u, v)=\frac{u . v}{\|u\|_{2}\|v\|_{2}}=\frac{\sum_{i=1}^{n} u_{i} v_{i}}{\sqrt{\sum_{i=1}^{n} u_{i}} \sqrt{\sum_{i=1}^{n} v_{i}}}
$$

\section{Euclidian Distance Similarity}

Let vector $u=\left(u_{1}, u_{2}, \ldots, u_{n}\right)^{T}$ and $v=\left(v_{1}, v_{2}, \ldots, v_{n}\right)^{T}$ are two $n$-dimensional vectors in vector space $\mathcal{R}^{\mathrm{n}}$, then Euclidian distance between these two vectors should be $\mathrm{L}_{2}$-norm of vector $(u-v)$ and is denoted by $\Delta(u, v)$ and defined using equation 6 . The Euclidian distance between these two vectors should be a real number between 0 and $\infty$. Since in our case, each tweet vector is a binary vector so the Euclidian distance value between each tweet-pair should be in range from 0 to $\sqrt{n}$. The smaller the distance shows both vectors are more similar and its larger value shows that both are most dissimilar. Equation 7 may be used get the similarity value between two tweet vectors using Euclidian distance method. Its value should be always between 0 and 1 .

$$
\begin{aligned}
& \Delta(u, v)=\|u-v\|_{2}=\sqrt{\sum_{i=1}^{n}\left(u_{i}-v_{i}\right)^{2}} \\
& \text { similarity }=\operatorname{sim}(u, v)=1-\frac{\Delta(u, v)}{\sqrt{n}}
\end{aligned}
$$

\section{PROPOSED TWEETS CLUSTERING AND ANALYSIS METHOD}

This section presents the functional details of different modules of our proposed tweets clustering and analysis method. The aim of proposed method is to partition the set of tweets into a number of clusters that represents various events. The functional detail of the various working modules of our proposed method is presented in figure 1. It starts by creating data set of tweets at local machine using tweet crawling module. The aim of features 
vector generation module is to convert each tweet into a binary vector which is used in similarity graph generation. Finally, with the help of similarity graph generation and graph clustering module we generate the similarity graph of the tweets and then cluster it using Markov clustering (MCL) graph clustering algorithm to partition it into a number of clusters, where each cluster represent a particular event. Following sub-section present the functional details of these modules.

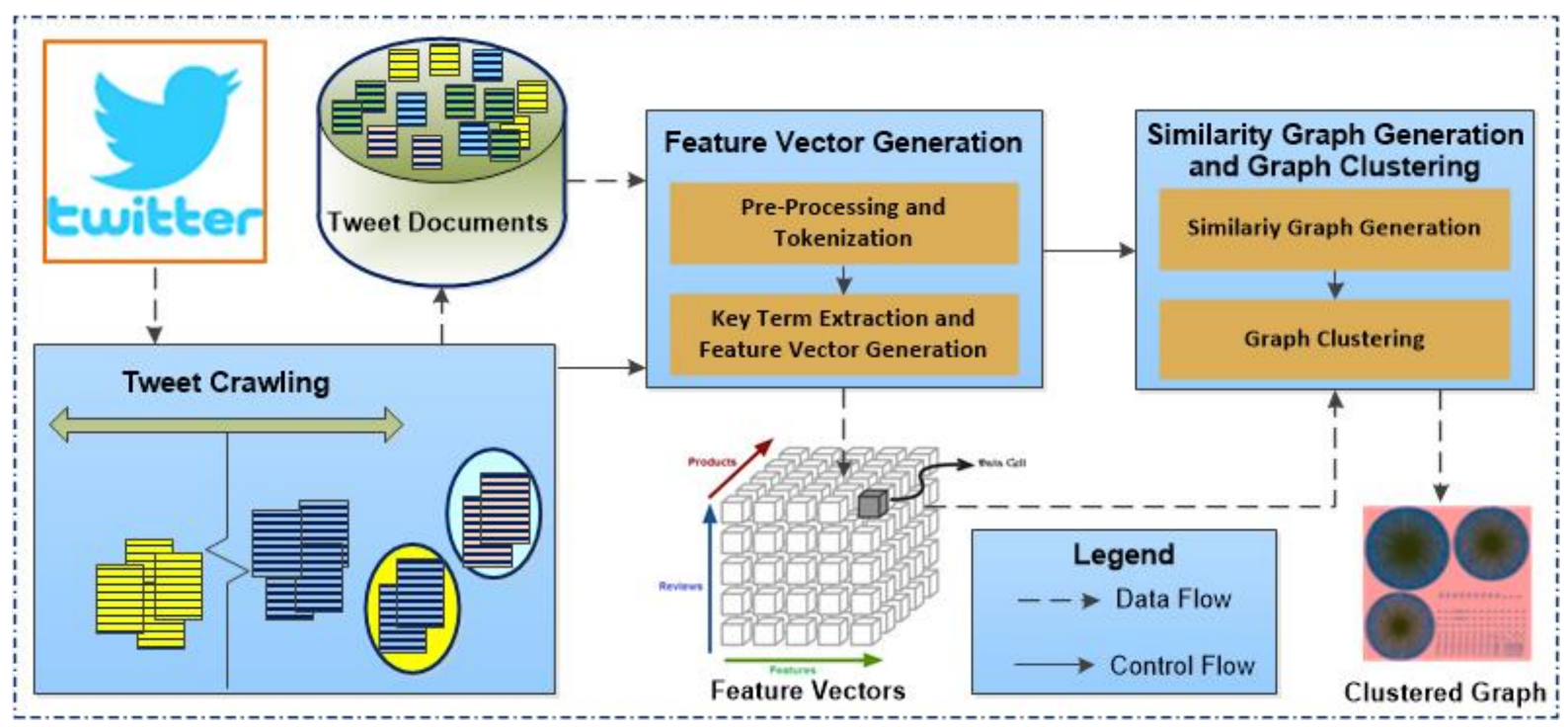

Figure 1. Functioning details of the proposed method

\section{A. Tweet Crawling}

In order to analyze the tweets, it is needed to create a data set of tweets on local machine. For this purpose, we have written a Java program using Twitter API to download the tweets from the Twitter. Our program downloads the various tweets and user related information along with tweet itself and stores them in a structured database table on local machine.

\section{B. Feature Vector Generation}

In order to generate the feature vectors corresponding to each tweet, first we have to clean the tweets by filtering the unwanted tokens like punctuation symbols, emoticons, special symbols, URLs etc. Thereafter, we convert each tweet into bag-of-words using 1-gram generation method. A word of more than two characters is valid if it is neither contains special characters nor is a stop-word, called candidate term.

To rank the candidate terms, we have used LDA technique. LDA is a probabilistic model used to determine latent topics from a document [25]. The input file for LDA is created as paragraphs of bag of words of candidate terms of tweets and first line of this file represent the number of paragraphs.

We have used JGibbLDA ${ }^{1}$ for execution of $L D A$ that generate $\Theta$ and $\Phi$ matrices, which is used to score the candidate terms. At the time of execution JGibbLDA, we have used the 0.1 and 0.5 values for parameters $\alpha$ and $\beta$ respectively and $q=100$ for number of topic. The score of each candidate term are calculated using equations 8 and 9 , where $\mathrm{k}$ is the number of paragraphs and $|\mathrm{s}[1]|$ is the total candidate terms in the ${ }^{\text {th }}$ paragraph. After ranking candidate term, we take top-n key terms for feature vector generation process. In [26], we had presented details of our key terms (aka key phrases) identification process.

$$
\begin{aligned}
\text { score }\left(t_{i}\right) & =\max _{j=1}^{q}\left\{\Phi_{j, i} \times \omega_{j}\right\} \\
\omega_{j} & =\sum_{l=1}^{k} \Theta_{l, j} \times|s[l]|
\end{aligned}
$$

Thereafter, we convert each tweet into an n-dimensional binary feature vector, who's each element should be either 0 or 1 depending upon absent and presence of corresponding key terms in the tweet.

${ }^{1} \mathrm{http} / /$ /jgibblda.sourceforge.net/ 


\section{Similarity Graph Generation and Graph Clustering}

The feature vectors corresponding to tweets are used to generate similarity graph (aka social network). In this graph each tweets are represent by nodes and weighted undirected edge between each node-pair is generated by calculating the similarity value between them. Between a node-pair an undirected weighted edge exist if corresponding similarity value is a positive quantity. The similarity value between each node-pair is calculated using cosine and Euclidian distance function.

The cosine similarity value between a node-pair is calculated using equation 5 . The algorithm 1 presents a formal way to generate the similarity graph using cosine similarity. The generated similarity graph should be undirected graph; therefore corresponding weighted adjacency matrix should be symmetric. Since cosine similarity of a vector with itself is 1 therefore every node of the corresponding similarity graph has a self loop.

Algorithm 1: generateSimilarityGraphusingCosineSimilarity $(\mathbf{V}, \mathbf{m}, \mathbf{n})$ : Similarity graph generation using cosine similarity.

Inputs: $2 \mathrm{D}$ array $\mathrm{V}$ of order $\mathrm{m} \mathrm{n}$ its $\mathrm{i}^{\text {th }}$ row represent the feature vector of $\mathrm{i}^{\text {th }}$ tweet in $\mathrm{n}$ dimensional vector space; $m$ number of tweets, $n$ number of features (key terms) of feature vectors of tweets.

Output: $2 \mathrm{D}$ array $\mathrm{W}$ of order $\mathrm{m} \mathrm{x} \mathrm{m}$ stores the similarity graph as weighted adjacency matrix.

1. genegateSimilarityGraphusingCosineSimilarity $(\mathrm{V}[][], \mathrm{m}, \mathrm{n})\{$

2. For $\mathrm{i}=1$ to $\mathrm{m}$ do \{

3. $\quad$ For $\mathrm{k}=1$ to $\mathrm{n}$ do \{

4. $\quad A[k]=V[i][k] ; \quad / / A$ is the feature vector of tweet-i

5. $\quad\}$

6. For $\mathrm{j}=1$ to $\mathrm{m}$ do \{

7. $\quad \operatorname{If}(\mathrm{i}>\mathrm{j})$ then $\left\{/ / \mathrm{W}\right.$ is symmetric matrix, $\therefore \mathrm{W}_{\mathrm{ij}}=\mathrm{W}_{\mathrm{ji}}$

8. $\quad W[i][j]=W[j][i]$;

9. 3

10. Else if $(\mathrm{i}==\mathrm{j})$ then $\left\{/ / \mathrm{W}_{\mathrm{ii}}=1\right.$

11. $\mathrm{W}[\mathrm{i}][\mathrm{i}]=1.0$;

12. $\}$

13. Else $\left\{/ /\right.$ calculate the cosine similarity between $V_{i} \& V_{j}$

14. For $\mathrm{k}=1$ to $\mathrm{n}$ do \{

15. $\quad B[k]=V[j][k] ; \quad / / B$ is the feature vector of the tweet- $\mathrm{j}$

16. $\}$

17. $W[\mathrm{i}][\mathrm{j}]=\operatorname{cosine}(\mathrm{A}, \mathrm{B})$; //using equation (5)

18. $\}$

19. $\}$

20. $\}$

21. Return W;

22. $\}$

To get the similarity graph using Euclidian distance method, first we calculate the distance between each node-pair using equation 6 . Since in our case, each tweet vector is an n-dimensional binary vector so the Euclidian distance value between each node-pair should in range from 0 to $\sqrt{n}$. Thereafter, we get the similarity value using Euclidian distance method between a node-pair using equation 7. The algorithm 2 presents a formal way to generate the similarity graph, of given set of tweets, using Euclidian distance method. It is also an undirected weighted graph, with each vertex having self loop. It is clear that Euclidian distance based similarity 
graph generating algorithm take more execution time and memory space in comparisons to cosine similarity based similarity graph generation.

Algorithm 2: generateSimilarityGraphusingOnEuclidianDistance(V, m, n): Similarity graph generation using Euclidian distance based similarity.

Inputs: $2 \mathrm{D}$ array $\mathrm{V}$ of order $\mathrm{m} \times \mathrm{n}$ its $\mathrm{i}^{\text {th }}$ row represent the feature vector of $\mathrm{i}^{\text {th }}$ tweet in $\mathrm{n}$ dimensional vector space; $m$ number of tweets, $n$ number of features (key terms) of feature vectors of tweets.

Output: 2D array $\mathrm{W}$ of order $\mathrm{m} \times \mathrm{m}$ stores the similarity graph as weighted adjacency matrix.

1. genegateSimilarityGraphusingEuclidianDistance(V[][], m, n)\{

2. For $\mathrm{i}=1$ to $\mathrm{m}$ do \{

3. For $\mathrm{k}=1$ to $\mathrm{n}$ do \{

4. $\quad \mathrm{A}[\mathrm{k}]=\mathrm{V}[\mathrm{i}][\mathrm{k}] ; \quad / / \mathrm{A}$ is the feature vector tweet- $\mathrm{i}$

5. $\}$

6. For $\mathrm{j}=\mathrm{i}+1$ to $\mathrm{m}$ do \{

7. For $\mathrm{k}=1$ to $\mathrm{n} \mathrm{do}\{$

8. $\quad B[k]=V[j][k] ; / / B$ is the feature vector of the tweet- $j$

9. $\}$

10. $\mathrm{d}[\mathrm{i}][\mathrm{j}]=\Delta(\mathrm{A}, \mathrm{B}) ; / /$ using equation $(6)$

11. \}

12. \}

13. For $\mathrm{i}=1$ to $\mathrm{m}$ do $\{/ /$ calculating similarity using Euclidian distance

14. For $\mathrm{j}=1$ to $\mathrm{m}$ do \{

15. If $(\mathrm{i}>\mathrm{j})$ then $\left\{/ / \mathrm{W}\right.$ is symmetric matrix, $\therefore \mathrm{W}_{\mathrm{ij}}=\mathrm{W}_{\mathrm{ji}}$

16. $\quad W[i][j]=W[j][i]$;

17. $\}$ Else if $(\mathrm{i}==\mathrm{j})$ then $\left\{/ / \mathrm{W}_{\mathrm{ii}}=1\right.$

18. $\quad W[\mathrm{i}][\mathrm{j}]=1$;

19. $\quad\}$ Else\{

20.

$$
\mathrm{W}[\mathrm{i}][\mathrm{j}]=1-(\mathrm{d}[\mathrm{i}][\mathrm{j}] / \sqrt{n}) ; / / \text { using equation } 7
$$

21. \}

22. \}

23. \}

24. Return $\mathrm{W}$;

25. \}

Once the similarity graph is generated for a tweet data set, MCL is used to partition the similarity graph into a number of directed sub-graphs (clusters), where each sub-graph represents a particular event. Each sub-graph in the partitioned graph has an attractor and other nodes belong to that sub-graph is attracted by it. The MCL algorithm is an iterative algorithm that partitions the graph using matrix expansion and inflation steps [27]. The MCL does not need the value of $\mathrm{k}$ (number of clusters); it requires inflation parameter $r$ whose value should be 
greater than 1. For less number of sub-graphs of larger size we take small value of $r$, whereas a large value of $r$ results more sub-graphs of smaller sizes.

\section{EXPERIMENTAL SETUP AND RESULTS}

In this section, we present evaluation results of our proposed tweet clustering and analysis method. For experimental evaluation purpose, we have created a data set of 5000 tweets by downloading tweets related to four different events - Uri attacks, Delhi assembly election, Union budget 2015, and Israel-Gaza conflict. Table 1 present the statistics about this tweet data set. Each tweet of this data set is converted into a 136-dimensional binary feature vector, for which we have taken top-136 key terms, as shown in Table 2. Next we generate the social network graph as a similarity graph using cosine similarity and Euclidian distance based similarity. In similarity graph generation process, we calculate the similarity between each pair of tweets based on their feature vectors.

TABLE I. TWEET DATA SET STATISTICS

\begin{tabular}{|c|c|c|c|c|c|c|c|}
\hline \multirow{2}{*}{$\begin{array}{c}\text { Tweet } \\
\text { Category }\end{array}$} & \multicolumn{4}{|c|}{ Tweets' Information } & \multicolumn{3}{c|}{ Authors' Information } \\
\cline { 2 - 8 } & $\begin{array}{c}\text { No. of } \\
\text { tweets }\end{array}$ & $\begin{array}{c}\text { Avg. no. of } \\
\text { hashtags }\end{array}$ & $\begin{array}{c}\text { Avg. no. of } \\
\text { URLs }\end{array}$ & $\begin{array}{c}\text { Avg. no. of } \\
\text { mentions }\end{array}$ & $\begin{array}{c}\text { Avg. no. of } \\
\text { followers }\end{array}$ & $\begin{array}{c}\text { Avg. no. of } \\
\text { friends }\end{array}$ & $\begin{array}{c}\text { Avg. no. of } \\
\text { tweets }\end{array}$ \\
\hline Uri Attacks & 1900 & 1.51 & 0.51 & 0.75 & 1595.73 & 735.45 & 28989.23 \\
\hline $\begin{array}{c}\text { Delhi } \\
\text { Assembly } \\
\text { Election }\end{array}$ & 900 & 0.29 & 0.48 & 1.02 & 2532.47 & 585.96 & 28513.22 \\
\hline $\begin{array}{c}\text { Union } \\
\text { Budget 2015 }\end{array}$ & 700 & 0.97 & 0.69 & 0.84 & 1485.59 & 968.85 & 27122.11 \\
\hline $\begin{array}{c}\text { Israel-Gaza } \\
\text { Conflict }\end{array}$ & 1500 & 1.31 & 0.36 & 0.89 & 1912.41 & 1112.84 & 17596.53 \\
\hline Total & $\mathbf{5 0 0 0}$ & $\mathbf{1 . 1 5}$ & $\mathbf{0 . 4 8}$ & $\mathbf{0 . 8 5}$ & $\mathbf{1 8 4 3 . 9 3}$ & $\mathbf{8 5 4 . 4 3}$ & $\mathbf{2 5 2 2 4 . 3 4}$ \\
\hline
\end{tabular}

Finally, social similarity graph is clustered using Markov CLustering (MCL) graph clustering algorithm. MCL is applied on the similarity graph generated by cosine similarity as well as Euclidian distance methods for values of $r$, ranging from 1.5 to 50.0, and finally 4.5 is considered as the optimal one for cosine similarity graph, as shown in Figure 3 the evaluation result of clustering for similarity graph generated by cosine similarity method. The clustered tweets graph on similarity graph generated by cosine similarity at $r=4.5$ is shown in Figure 2, in which blue v-shapes, purple circles, green triangles, and red squares are used to represent Uri Attacks, Delhi assembly election, union budget 2015, and Israel-Gaza conflict events respectively. It is examine from this figure that besides four bigger sub-graphs each corresponding to an event, there are some isolated nodes. On manual analysis of content of the tweets corresponding to these isolated nodes, we gets that it does not have enough content to represent the events under consideration and they can be considered as outliers.

TABLE II. TOP 136 KEY TERMS AND THEIR RANK SCORES

\begin{tabular}{|l|l|l|l|l|l|l|l|}
\hline Key term & $\begin{array}{l}\text { Rank } \\
\text { score }\end{array}$ & Key term & $\begin{array}{l}\text { Rank } \\
\text { score }\end{array}$ & Key term & $\begin{array}{l}\text { Rank } \\
\text { score }\end{array}$ & Key term & $\begin{array}{l}\text { Rank } \\
\text { score }\end{array}$ \\
\hline uriattacks & 1224.38 & fatwa & 59.87 & Mother & 31.27 & military & 22.51 \\
\hline palestine & 537.55 & blame & 59.19 & Kiski & 31.13 & arun & 22.04 \\
\hline unitedagainstpak & 460.38 & free & 57.61 & World & 30.90 & finance & 22.04 \\
\hline gaza & 433.01 & stop & 54.56 & Illegal & 30.14 & tax & 21.45 \\
\hline israel & 360.53 & election & 53.71 & President & 30.14 & budget2015 & 21.45 \\
\hline whereisrss & 338.46 & reasons & 53.71 & Respect & 29.69 & injured & 20.98 \\
\hline delhi & 293.20 & uripayback & 52.65 & Watch & 28.90 & anti & 20.98 \\
\hline budget & 277.73 & hammas & 52.27 & Pray & 28.61 & vajpayee & 20.98 \\
\hline aap & 254.20 & people & 52.27 & Minister & 28.51 & banks & 20.22 \\
\hline pakistan & 233.16 & nation & 51.86 & Grand & 28.11 & financing & 20.22 \\
\hline india & 195.95 & conflict & 50.74 & Results & 27.71 & boycott & 20.19 \\
\hline union & 186.04 & introspect & 48.92 & Afghanistan & 26.52 & terrorism & 20.19 \\
\hline actagainstpak & 171.41 & pay & 47.11 & Victory & 26.34 & terror & 20.19 \\
\hline kejriwal & 170.04 & army & 46.32 & Uphold & 26.32 & afford & 20.19 \\
\hline urimartyrs & 144.49 & war & 46.16 & Corporate & 26.32 & mrsgandhi & 19.40 \\
\hline israeli & 143.07 & civilians & 45.40 & Responsibility & 26.32 & serious & 19.40 \\
\hline maunmodisarkar & 142.90 & occupation & 45.40 & Syria & 26.32 & industry & 19.10 \\
\hline
\end{tabular}




\begin{tabular}{|l|c|l|l|l|l|l|l|}
\hline wakeupmodi & 127.07 & uri & 44.73 & Terrorists & 25.56 & death & 17.93 \\
\hline hamas & 120.94 & terrorstatepak & 43.94 & Chief & 24.98 & protest & 17.93 \\
\hline bjp & 115.98 & soldier & 43.15 & Sarkar & 24.98 & dies & 17.81 \\
\hline terrorist & 111.24 & human & 43.11 & Post & 24.94 & innocent & 17.17 \\
\hline modi & 97.78 & action & 42.36 & Killing & 24.94 & speech & 17.02 \\
\hline palestinian & 91.94 & un & 40.06 & Bastards & 24.94 & proud & 17.02 \\
\hline bedi & 88.61 & pm & 39.98 & Support & 24.80 & ashamed & 17.02 \\
\hline kiran & 85.19 & arvindkejriwal & 37.29 & Freedom & 24.15 & govt & 16.76 \\
\hline soldiers & 78.78 & polls & 37.29 & Fighters & 24.15 & rail & 16.75 \\
\hline unionbudget2015 & 77.29 & attack & 35.23 & Dead & 24.04 & kids & 16.40 \\
\hline gazaunderattack & 72.87 & peace & 34.72 & Media & 24.04 & protect & 16.40 \\
\hline martyr & 70.86 & hospital & 33.95 & Netanyahu & 24.04 & nationalism & 16.23 \\
\hline arvind & 68.77 & kill & 33.95 & Russia & 23.36 & nawazsharif & 16.23 \\
\hline dilli & 67.40 & freepalestine & 33.19 & Pmoindia & 23.36 & jaitley & 16.16 \\
\hline killed & 63.71 & jews & 33.19 & Israelpalestine & 23.27 & secretary & 16.08 \\
\hline loss & 61.24 & homage & 32.86 & Keeping & 22.56 & superbudget & 15.58 \\
\hline children & 59.90 & rockets & 31.67 & Appeal & 22.56 & won & 15.40 \\
\hline
\end{tabular}

We have evaluated the proposed method in term of $F_{P}$ and $F_{B}$, where $F_{P}$ is the harmonic mean of purity and inverse purity, and $\mathrm{F}_{\mathrm{B}}$ is the harmonic mean of $B$-cubed precision and $B$-cubed recall.

Purity: The purity evaluation metric is defined using equation 10 , where $C_{i}$ is a sub-graph contains $i^{\text {th }}$ node (tweet) in partitioned graph, $\mathrm{L}_{\mathrm{j}}$ is the actual sub-graph for $\mathrm{j}^{\mathrm{th}}$ node (tweet), and $\mathrm{n}$ is the size of data set.

$$
\text { purity }=\sum_{i} \frac{\left|C_{i}\right|}{n} \times \operatorname{maxPrecision}\left(C_{i}, L_{j}\right)
$$

Inverse Purity: The inverse purity evaluation metric is defined using equation 11 , where $\mathrm{C}_{\mathrm{i}}$ is a sub-graph contains $i^{\text {th }}$ node (tweet) in partitioned graph, $L_{j}$ is the actual sub-graph for $j^{\text {th }}$ node (tweet), and $n$ is the size of data set.

$$
\text { inversePurity }=\sum_{i} \frac{\left|L_{i}\right|}{n} \times \operatorname{maxPrecision}\left(L_{i}, C_{j}\right)
$$

B-Cubed Precision: The B-cubed precison is defined as average of precision of individual nodes. The precision of $i^{\text {th }}$ node is calculated using equation 12 , where $C_{i}$ is a sub-graph contains $i^{\text {th }}$ node (tweet) in partitioned graph, $\mathrm{L}_{\mathrm{i}}$ is the actual sub-graph for $\mathrm{i}^{\text {th }}$ node (tweet).

$$
\operatorname{precision}(i)=\frac{\left|C_{i} \cap L_{i}\right|}{\left|C_{i}\right|}
$$

B-Cubed Recall: The B-cubed recall is defined as average of recall of individual nodes. The recall of $\mathrm{i}^{\text {th }}$ node is calculated using equation 13 , where $C_{i}$ is a sub-graph contains $i^{\text {th }}$ node (tweet) in partitioned graph, $L_{i}$ is the actual sub-graph for $\mathrm{i}^{\text {th }}$ node (tweet).

$$
\operatorname{recall}(i)=\frac{\left|C_{i} \cap L_{i}\right|}{\left|L_{i}\right|}
$$

For evaluation purpose, we have labelled each nodes of the partitioned graph as " $U A$ ”, “DE”, “UB”, and " $G$ ” which are corresponding to tweets "Uri Attacks", “ Delhi assembly election”, "union budget 2015”, and "Israel-Gaza conflict" respectively. To get the evaluation metric for partitioned graph, we have written a Java program that takes the name of file containing the partitioned graph and number of total sub-graphs as input and 
generate evaluation metric values as output. Table 3 present the evaluation result for clustering on similarity graph generated by cosine similarity and Euclidian distance based similarity, for different inflation parameter $r$. Figure 3 is a graphical form of the evaluation results shown in Table 3 . It is determined from these table and figure that the values of both $\mathrm{F}_{\mathrm{P}}$ and $\mathrm{F}_{\mathrm{B}}$ parameters is highest for $r=4.5$ for clustering using MCL algorithm on similarity graph generated by cosine similarity method. The MCL graph clustering algorithm generate only one cluster for different value of inflation parameter $r$ range from 1.5 to 90 on similarity graph generated by Euclidian distance based similarity. Therefore, the $F_{P}$ and $F_{B}$ in this case is constant and much lower than that are for cosine similarity graph. This study show that for analysis of tweets, the cosine similarity techniques is significant and Euclidian distance based similarity graph is not suitable.

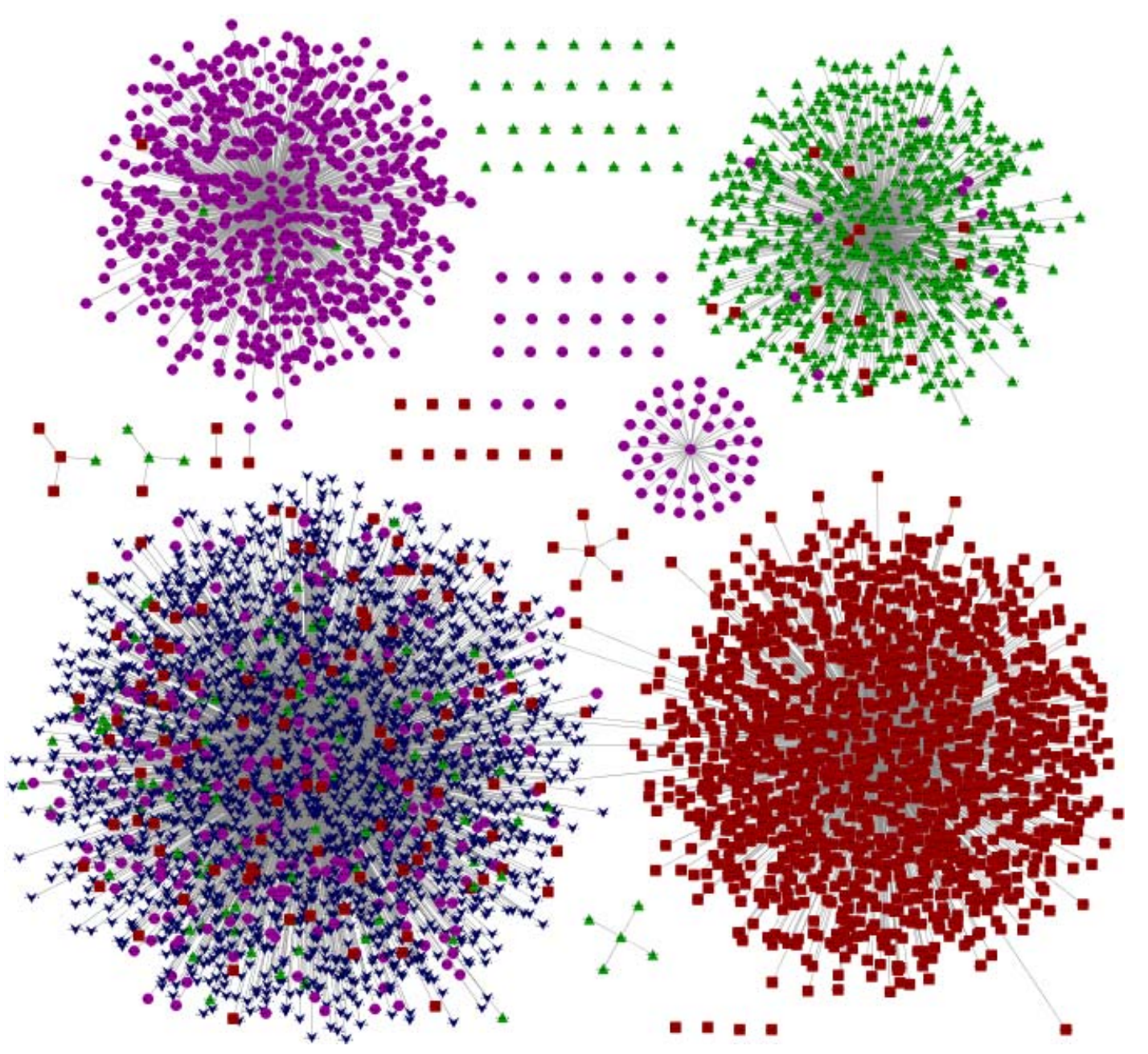

Figure 2. Clustered tweets graph using MCL with $\mathrm{r}=4.5$ on cosine similarity graph

TABLE III. EVALUATION RESULT OF THE CLUSTERED GRAPH FOR DIFFERENT R VALUES

\begin{tabular}{|c|c|c|c|c|c|c|c|c|c|c|}
\hline $\mathbf{r}$ & $\begin{array}{c}\text { No. of } \\
\text { connected } \\
\text { components }\end{array}$ & $\begin{array}{c}\text { No. of } \\
\text { nodes }\end{array}$ & $\begin{array}{c}\text { No. of } \\
\text { isolated } \\
\text { nodes }\end{array}$ & Purity & $\begin{array}{c}\text { Inverse } \\
\text { Purity }\end{array}$ & FP & $\begin{array}{c}\text { Average } \\
\text { B-Cube } \\
\text { precision }\end{array}$ & $\begin{array}{c}\text { Average } \\
\text { B-Cube } \\
\text { recall }\end{array}$ & FB \\
\hline \multicolumn{8}{|c|}{ Similarity Graph using Cosine Similarity } \\
\hline 1.5 & 60 & 5000 & 59 & 0.3918 & 0.9882 & 0.5611 & 0.2972 & 0.9767 & 0.4558 \\
\hline 2.5 & 66 & 5000 & 61 & 0.6644 & 0.9584 & 0.7848 & 0.5549 & 0.9227 & 0.6930 \\
\hline 3.5 & 69 & 5000 & 61 & 0.7742 & 0.8912 & 0.8286 & 0.6697 & 0.8431 & 0.7464 \\
\hline $\mathbf{4 . 5}$ & $\mathbf{7 3}$ & $\mathbf{5 0 0 0}$ & $\mathbf{6 2}$ & $\mathbf{0 . 9 1 3 4}$ & $\mathbf{0 . 8 8 8 0}$ & $\mathbf{0 . 9 0 0 5}$ & $\mathbf{0 . 8 4 6 9}$ & $\mathbf{0 . 8 1 6 2}$ & $\mathbf{0 . 8 3 1 3}$ \\
\hline 5.2 & 75 & 5000 & 64 & 0.9228 & 0.8616 & 0.8912 & 0.8616 & 0.7717 & 0.8142 \\
\hline 5.5 & 75 & 5000 & 64 & 0.9254 & 0.8546 & 0.8886 & 0.8656 & 0.7624 & 0.8108 \\
\hline 6.5 & 80 & 5000 & 68 & 0.9326 & 0.8458 & 0.8871 & 0.8775 & 0.7493 & 0.8083 \\
\hline 7.0 & 81 & 5000 & 68 & 0.9346 & 0.8240 & 0.8758 & 0.8807 & 0.7227 & 0.7939 \\
\hline 10.0 & 86 & 5000 & 69 & 0.9490 & 0.7784 & 0.8553 & 0.9049 & 0.6858 & 0.7803 \\
\hline
\end{tabular}




\begin{tabular}{|l|c|c|c|c|c|c|c|c|c|}
\hline 20.0 & 92 & 5000 & 69 & 0.9564 & 0.6738 & 0.7906 & 0.9179 & 0.5902 & 0.7184 \\
\hline 30.0 & 104 & 5000 & 72 & 0.9584 & 0.6170 & 0.7507 & 0.9216 & 0.5251 & 0.6691 \\
\hline 50.0 & 114 & 5000 & 73 & 0.9680 & 0.5520 & 0.7031 & 0.9432 & 0.4199 & 0.5811 \\
\hline \multicolumn{7}{|c|}{ Similarity Graph using Euclidian Distance Similarity } \\
\hline 1.5 & 1 & 5000 & 0 & 0.3800 & 1.0000 & 0.5507 & 0.2864 & 1.0000 & 0.4453 \\
\hline 2.5 & 1 & 5000 & 0 & 0.3800 & 1.0000 & 0.5507 & 0.2864 & 1.0000 & 0.4453 \\
\hline 3.5 & 1 & 5000 & 0 & 0.3800 & 1.0000 & 0.5507 & 0.2864 & 1.0000 & 0.4453 \\
\hline 4.5 & 1 & 5000 & 0 & 0.3800 & 1.0000 & 0.5507 & 0.2864 & 1.0000 & 0.4453 \\
\hline 5.2 & 1 & 5000 & 0 & 0.3800 & 1.0000 & 0.5507 & 0.2864 & 1.0000 & 0.4453 \\
\hline 5.5 & 1 & 5000 & 0 & 0.3800 & 1.0000 & 0.5507 & 0.2864 & 1.0000 & 0.4453 \\
\hline 6.5 & 1 & 5000 & 0 & 0.3800 & 1.0000 & 0.5507 & 0.2864 & 1.0000 & 0.4453 \\
\hline 7.0 & 1 & 5000 & 0 & 0.3800 & 1.0000 & 0.5507 & 0.2864 & 1.0000 & 0.4453 \\
\hline 10.0 & 1 & 5000 & 0 & 0.3800 & 1.0000 & 0.5507 & 0.2864 & 1.0000 & 0.4453 \\
\hline 20.0 & 1 & 5000 & 0 & 0.3800 & 1.0000 & 0.5507 & 0.2864 & 1.0000 & 0.4453 \\
\hline 30.0 & 1 & 5000 & 0 & 0.3800 & 1.0000 & 0.5507 & 0.2864 & 1.0000 & 0.4453 \\
\hline 50.0 & 1 & 5000 & 0 & 0.3800 & 1.0000 & 0.5507 & 0.2864 & 1.0000 & 0.4453 \\
\hline 90.0 & 1 & 5000 & 0 & 0.3800 & 1.0000 & 0.5507 & 0.2864 & 1.0000 & 0.4453 \\
\hline
\end{tabular}

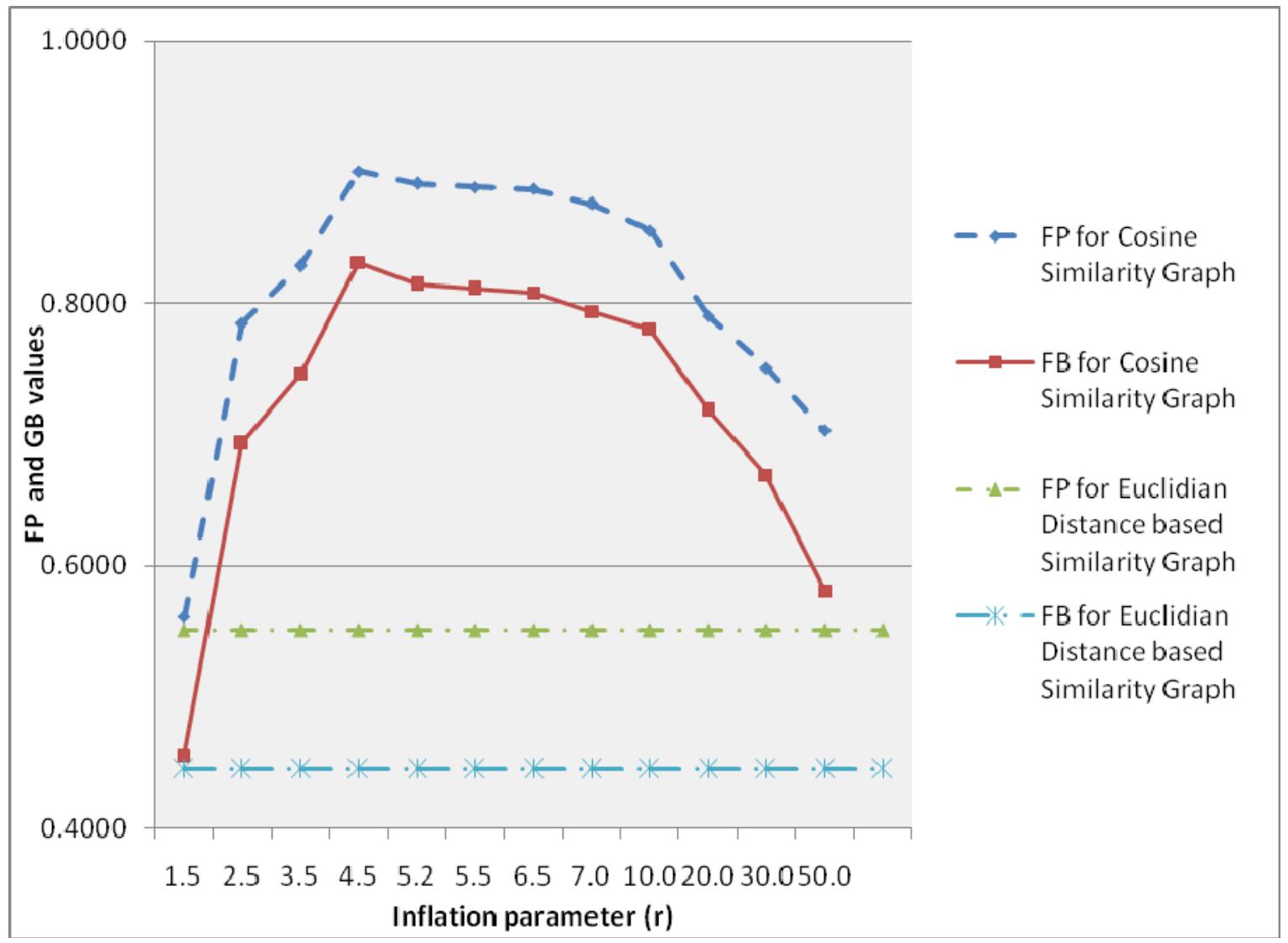

Figure 3. Visualization of $F_{P}$ and $F_{B}$ measures for different inflammation parameter (r) values

\section{CONCLUSION}

In this paper, we have presented a content-based tweets analysis method to identify various kind of events discussed over Twitter. We have used LDA to identify key terms from tweets and represent them as a feature vector for social graph generation. Thereafter, a graph-based algorithm, MCL, is applied which performs a random walk over the social graph to identify dense regions, i.e., clusters. We have also presented a comparative analysis of Cosine similarity and Euclidean distance measures to see their roles in social graph generation and cluster identification. It can be observed from Figure 3 that Cosine similarity performs better than Euclidean distance measure in terms of both $F_{P}$ and $F_{B}$ measures for all values of the inflammation parameter $(r)$. Another observation which can be made from Figure 3 is that the values of $F_{P}$ and $F_{B}$ measures are constant in case of Euclidean distance for all values of $r$, whereas, in case of Cosine similarity measure, the values of $F_{P}$ and $F_{B}$ measures are increasing with increasing value of $r$ until $r=4.5$ at which the actual number of clusters are obtained, thereafter it starts decreasing when clusters start splitting into smaller clusters due to larger $r$ values. 
On the other hand, after analyzing the functioning details of the Markov Clustering, it is found that the number of clusters to be identified by the Markov Clustering is determined by the value of the inflation parameter $(r)$, i.e., number of clusters are less for smaller value of $r$, and more for the larger values of $r$. Hence, the Cosine similarity measure is able to mimic the true functioning of the Markov Clustering. As a result, it can be concluded that Cosine similarity seems more effective in comparison to the Euclidean distance to capture and model the underlying social structure of the given data set. Working towards a hybrid approach, exploiting both content-based and structural features of data sets for event identification seems one of the promising future directions of research.

\section{REFERENCES}

[1] N. Azam, Jahiruddin, M. Abulaish, and N. Al-H. Haldar, "Twitter Data Mining for Events Classification and Analysis", In Proceedings of the 2nd International Conference on Soft Computing and Machine Intelligence (ISCMI'15), Hong Kong, IEEE CPS, Nov. 23-24, 2015, pp. 79-83.

[2] Mohd. Fazil, and Muhammad Abulaish, Identifying Active, Reactive, and Inactive Targets of Socialbots in Twitter, In Proceedings of the IEEE/WIC/ACM International Conference on Web Intelligence (WI), ACM, Lepzig, Germany, pp. 573-580, August 23-26, 2017.

[3] Mohd Fazil and Muhammad Abulaish, Why a Socialbot is Effective in Twitter? A Statistical Insight, In Proceedings of the 9th International Conference on Communication Systems and Networks (COMSNETS), Social Networking Workshop, Bengaluru, India, pp. 562-567, January 4-8, 2017.

[4] Muhammad Abulaish and Sajid Y. Bhat, Classifier Ensembles using Structural Features for Spammer Detection in Online Social Networks, Foundations of Computing and Decision Sciences, Vol. 40, Issue 2, Poznan University of Technology, Poland, pp. 89-105, 2015.

[5] Sajid Y. Bhat and Muhammad Abulaish, Communities against Deception in Online Social Networks, Computer Fraud and Security, 2014(2), Elsevier, ISSN: 1361-3723, pp. 8-16, Feb. 2014.

[6] Tarique Anwar and Muhammad Abulaish, A social graph based text mining framework for chat log investigation, Digital Investigation, Vol. 11, Issue 4, Elsevier, pp. 349-362, 2014.

[7] Sajid Y. Bhat, Muhammad Abulaish, and Abdulrahman A. Mirza, Spammer Classification using Ensemble Methods over Structural Social Network Features, In Proceedings of the 14th IEEE/WIC/ACM International Conference on Web Intelligence (WI'14), Warsaw, Poland, pp. 454-458, Aug. 11-14, 2014.

[8] J. Chung and E. Mustafaraj, "Can collective sentiment expressed on twitter predict political elections?" in Proceedings of the TwentyFifth AAAI Conference on Artificial Intelligence, 2011, pp. 170-171.

[9] M. Cheong and V. Lee, "A study on detecting patterns in twitter intratopic user and message clustering," in Proceedings of the 2010 20th International Conference on Pattern Recognition, 2010, pp. 3125-3128.

[10] C. G. Akcora, M. A. Bayir, M. Demirbas, and H. Ferhatosmanoglu, "Identifying breakpoints in public opinion," in Proceedings of the First Workshop on Social Media Analytics, 2010, pp. 62-66.

[11] J. Bollen, H. Mao, and X.-J. Zeng, "Twitter mood predicts the stock market," Journal of Computational Science, vol. 2, no. 1, pp. 1-8, 2011.

[12] M. Thelwall, K. Buckley, and G. Paltoglou, "Sentiment in twitter events," Journal of the American Society for Information Science and Technology, vol. 62, no. 2, pp. 406-418, 2011.

[13] M. Cha, H. Haddadi, F. Benevenuto, and K. P. Gummadi, "Measuring user influence in twitter: The million follower fallacy," in Proceedings of the Fourth International AAAI Conference on Weblogs and Social Media, 2010, pp. 10-17.

[14] A. Willis, A. Fisher, and I. Lvov, "Mapping networks of influence: Tracking Twitter conversations through time and space," Journal of Audience and Reception Studies, vol. 12, no. 1, pp. 594-530, 2015.

[15] A. Pak and P. Paroubek, "Twitter as a corpus for sentiment analysis and opinion mining," in Proceedings of the Seventh conference on International Language Resources and Evaluation (LREC'10), 2010, pp. 1320-1326.

[16] A. Go, L. Huang, and R. Bhayani, "Twitter sentiment analysis," Stanford University, Stanford, California, USA, CS224N - Final Project Report, 2009.

[17] J. Spencer and G. Uchyigit, "Sentimentor: Sentiment Analysis on Twitter Data," in Proceedings of the 1st International Workshop on Sentiment Discovery from Affective Data, Bristol, United Kingdom, 2012, pp. 56-66.

[18] A. Go, R. Bhayani, and L. Huang, "Twitter sentiment classification using distant supervision," Processing 150(12), pp. 1-6, 2009.

[19] H. Becker, M. Naaman, and L. Gravano, "Learning similarity metrics for event identification in social media," in Proceedings of the third ACM international conference on Web search and data mining, 2010, pp. 291-300.

[20] T. Sakaki, M. Okazaki, and Y. Matsuo, "Earthquake shakes twitter users: Real-time event detection by social sensors," in Proceedings of the 19th international conference on World wide web, 2010, pp. 851-860.

[21] H. Becker, M. Naaman, and L. Gravano, "Beyond trending topics: Real-world event identification on twitter," in Proceedings of the Fifth International AAAI Conference on Weblogs and Social Media, 2011, pp. 438-441.

[22] Sajid Y. Bhat and Muhammad Abulaish, Analysis and Mining of Online Social Networks - Emerging Trends and Challenges, WIREs Data Mining and Knowledge Discovery, 3(6), John Wiley and Sons, ISSN: 1942-4787, pp. 408-444, Nov/Dec 2013.

[23] Peter J. Olver. 2008. Numerical Analysis Lecture Note. Retrieved on 18.03.2017 from http://www-users.math.umn.edu/ olver/num /lnn.pdf.

[24] E.W. Weisstein. 2002. Vector Norm. Wolfram MathWorld. Retrieved on 18.03.2017 from http://mathworld.wolfram.com/ VectorNorm.html.

[25] D. M. Blei, A. Y. Ng, and M. I. Jordan, "Latent dirichlet allocation,” Journal of Machine Learning Research, vol. 3, no. 4-5, pp. 9931022,2003

[26] M. Abulaish, Jahiruddin, and L. Dey, "Deep text mining for automatic keyphrase extraction from text documents," Journal of Intelligent Systems, vol. 20, no. 4, pp. 327-351, 2011.

[27] S. van Dongen, "Graph clustering by flow simulation," Ph.D. Thesis, University of Utrecht, Utrecht, Netherlands, 2000. 